\title{
A CHARACTERIZATION OF SUBNORMAL OPERATORS
}

\author{
by ALAN LAMBERT $\dagger$
}

(Received 18 October, 1982)

Introduction. In this note a characterization of subnormality of operators on Hilbert space is given. The characterization is in terms of a sequence of polynomials in the operator and its adjoint reminiscent of the binomial expansion in commutative algebras. As such no external Hilbert spaces are needed, nor is it necessary to introduce forms dependent on arbitrary sequences of vectors from the Hilbert space.

Subnormality. Let $H$ be a complex Hilbert space and let $B(H)$ be the algebra of all bounded linear operators on $H$. The operator $A$ in $B(H)$ is said to be subnormal if there is a normal operator on a space containing $H$ which leaves $H$ invariant and whose restriction to $H$ is $A$. There are several characterizations of subnormal operators; e.g., [1], [2], [5]. In most cases the characterizations involve representations of $A$ on spaces different from or, as in the classic characterization of J. Bram, assertions about large families of multilinear forms. In this note we offer a characterization in terms of a sequence of polynomials in $A$ and $A^{*}$. The motivation for this characterization may be found in the following lemma.

Lemma. Let $A$ be a subnormal operator on $H$ with normal extension $N$ on $K \geqslant H$. Suppose that $\operatorname{Re} A \geqslant 0$ and $\operatorname{Re} N \geqslant 0$. Then for every $n \geqslant 0, \sum_{k=0}^{n}\left(\begin{array}{l}n \\ k\end{array}\right) A^{* n-k} A^{k} \geqslant 0$.

Proof. Let $P$ be the orthogonal projection of $K$ onto $H$. We have for $n \geqslant 0$,

$$
\sum_{k=0}^{n}\left(\begin{array}{l}
n \\
k
\end{array}\right) N^{* n-k} N^{k}=(2 \operatorname{Re} N)^{n} \geqslant 0
$$

hence

$$
\sum_{k=0}^{n}\left(\begin{array}{l}
n \\
k
\end{array}\right) A^{* n-k} A^{k}=\sum_{k=0}^{n}\left(\begin{array}{l}
n \\
k
\end{array}\right)\left(P N^{* n-k} P\right)\left(P A^{k}\right) P=P(2 \operatorname{Re} N)^{n} P \geqslant 0 .
$$

Now, for any operator $A$ define $S_{n}=S_{n}(A)$ to be $\sum_{k=0}^{n}\left(\begin{array}{l}n \\ k\end{array}\right) A^{* n-k} A^{k}$ and note that $S_{n}$ is self-adjoint. The sequence $\left\{S_{n}\right\}$ may be obtained in the following way. Let $\left\{T_{t}\right\}_{1 \geq 0}$ be the uniformly continuous semigroup $T_{t}=e^{-t A}$. One sees that $S_{1}=2 \operatorname{Re} A$ and $S_{n+1}=$ $2 \operatorname{Re}\left(A^{*} S_{n}\right)$. It then follows easily that, for each $x$ in $H$,

$$
\left(d^{n} / d t^{n}\right)\left(\left\|T_{t} x\right\|^{2}\right)=(-1)^{n}\left(S_{n} T_{t} x, T_{t} x\right)
$$

$\dagger$ This work was undertaken while the author was a visiting scientist at the Weizmann Institute of Science, Rehovot, Israel. The author gratefully acknowledges the Institute's support.

Glasgow Math. J. 25 (1984) 99-101. 
and so

$$
\left(S_{n} x, x\right)=\left.(-1)^{n}\left(d^{n} / d t^{n}\right)\left(\left\|T_{t} x\right\|^{2}\right)\right|_{t=0} .
$$

In particular $\left\{S_{n}\right\}$ is a sequence of positive operators if and only if for every $x$ in $H$, the mapping $t \rightarrow\left\|T_{t} x\right\|^{2}$ is completely monotone (see [6; Cptr. IV]). Now it is known that the $C^{\infty}$ function $f$ on $[0, \infty)$ is completely monotone if and only if there is a Borel measure $\mu$ on $[0, \infty)$ such that $f(t)=\int_{0}^{\infty} e^{-t s} d \mu(s)$. We have established the following result.

Proposttion. $\left\{S_{n}(A)\right\}$ is a sequence of positive operators if and only if for each $x \neq 0$ in $H$ there is a Borel measure $\mu=\mu_{x}$ on $[0, \infty)$ such that $\left\|e^{-t A} x\right\|^{2}=\int_{0}^{\infty} e^{-t s} d \mu_{x}(s)$.

Before obtaining the promised characterization of subnormality, we associate with the operator $A$ a family of weighted translation semigroups. A semigroup $\left\{E_{\imath}\right\}$ on $L^{2}([0, \infty), d s)$ is a weighted translation semigroup if there is a strictly positive continuous function $\varphi$ on $[0, \infty)$ such that

$$
\left(E_{\mathrm{t}} f\right)(s)= \begin{cases}(\varphi(s) / \varphi(s-t)) f(s-t) & (s \geqslant t), \\ 0 & (s<t) .\end{cases}
$$

These semigroups are discussed in detail in [3] and [4]. It is shown in [3] that $E_{t}$ is strongly continuous if and only if there is an $M>0$ and a real number $w$ such that, for all $t \geqslant 0$, $\sup \varphi(s+t) / \varphi(s) \leqslant M e^{w t}$.

$s$ Also, $\left\{E_{t}\right\}$ is a semigroup of subnormal operators if and only if there is a Borel measure $\rho$ on $[0, \infty)$ such that $\varphi^{2}(t)=\int_{0}^{\infty} e^{-t s} d \rho(s)([4])$.

If $\left\{B_{t}\right\}$ is any strongly continuous semigroup on $H$ and $\operatorname{Ker} B_{t}=0$ for all $t \geqslant 0$, fix $x \neq 0$ and let $\varphi(t)=\left\|B_{t} x\right\|$. Then since $B_{t}$ is strongly continuous, $\left\|B_{t}\right\| \leqslant M e^{w t}$ for some $M$ and $w$. Thus $\varphi(s+t) / \varphi(s)=\left\|B_{t} B_{s} x\right\| /\left\|B_{s} x\right\| \leqslant M e^{w t}$. In particular the weighted translation semigroup $\left\{E_{t}^{x}\right\}$ corresponding to $\varphi$ is strongly continuous.

THEOREM. Let $A$ be in $B(H)$ with $\operatorname{Re} A \geqslant 0$. Then $A$ is subnormal if and only if $S_{n}(A) \geqslant 0$ for each $n \geqslant 0$.

Proof. The lemma above (together with elementary properties of the minimal normal extension of a subnormal operator) shows that if $A$ is subnormal, then each $S_{n} \geqslant 0$. Suppose now that each $S_{n} \geqslant 0$. Let $T_{t}=e^{-t A}$. Then we know that for each $x \neq 0$, the mapping $t \rightarrow\left\|T_{t} x\right\|^{2}$ is completely monotone and thus each of the weighted translation semigroups $\left\{E_{t}^{x}\right\}$ corresponding to $\left\|T_{t} x\right\|$ is subnormal. Fix $x \neq 0$ in $H$. We have seen that there is a Borel measure $d \rho$ on $[0, \infty)$ such that $\left\|T_{t}^{n} x\right\|^{2}=\left\|T_{n t} x\right\|^{2}=\int_{0}^{\infty} e^{-n t s} d \rho(s)$.

Now it is shown in [5] that if $\operatorname{Ker} B=0$, then $B$ is subnormal if and only if for each $x \neq 0$ there is a Borel measure $\mu$ on $[0,\|B\|]$, such that $\left\|B^{n} x\right\|^{2}=\int_{0}^{\|B\|} s^{n} d \mu(s)$. After a suitable change of variables we see that for fixed $t, T_{t}$ is subnormal and so $\left\{T_{t}\right\}$ is a subnormal semigroup. But it is known that the generator of a subnormal semigroup is subnormal. Thus $A$ is subnormal, completing the proof. 
Remark. The assumption that $\operatorname{Re} A \geqslant 0$ is of only technical importance since $A$ and $A+\alpha I$ subnormally stand or fall together. Without this assumption the main result must be rephrased as: $A$ is subnormal if and only if for some $\alpha, S_{n}(A+\alpha I) \geqslant 0$ for all $n$; or equivalently, for some $\beta, \sum_{k=0}^{n}\left(\begin{array}{l}n \\ k\end{array}\right) \beta^{k} S_{n-k} \geqslant 0$ for all $n$.

\section{REFERENCES}

1. J. Bram, Subnormal operators, Duke Math. J., 22 (1955), 75-94.

2. M. R. Embry, A generalization of the Halmos-Bram criterion for subnormality, Acta Sci. Math. (Szeged), 35 (1973), 61-64.

3. M. R. Embry and A. Lambert, Weighted translation semigroups, Rocky Mountain J. Math., 7 (1977), 333-344.

4. M. R. Embry and A. Lambert, Subnormal weighted tranlsation semigroups, I. Functional Analysis 24 (1977), 268-275.

5. A. Lambert, Subnormality and weighted shifts, J. London Math. Soc., (2) 14 (1976), 476-480.

6. D. W. Widder, The Laplace transform, (Princeton Univ. Press, Princeton, N.J., 1946).

The Weizmann Institute of Science

REHOVOT, ISRAEL

Present address:

University of North Carolina

Charlotte

North Carolina 28223

U.S.A. 